\title{
On Thermal Stability Analysis for a Reacting Slab
}

\author{
Bakari Hamza ${ }^{1}$, Es tomih S. Massawe ${ }^{1, *}$, Charles W. Mahera ${ }^{1}$, Oluwole D. Makinde ${ }^{2}$ \\ ${ }^{1}$ Mathematics Department, University of Dar es Salaam, P. O. Box 35062, Tanzania \\ ${ }^{2}$ Faculty of Engineering, Cape Peninsula University of Technology, P. O. Box 1906, Bellville 7535, South Africa
}

\begin{abstract}
In this chapter, thermal stability analysis of the steady state exothermic chemical reaction in a slab of combustible material is investigated in the present of convective heat loss to the ambient. The nonlinear differential equations governing the system are obtained and solved using perturbation technique together with a special type of Hermite-Padé series summation and improvement method. The effects of various embedded parameters on the temperature profile and thermal stability of the system are presented graphically and discussed quantitatively. The possibility of thermal runaway phenomena was shown and the corresponding thermal criticality values were obtained and illustrated on a bifurcation diagram. The results reveal the thermal stability criticality as well as the effects of various embedded parameters on the system.
\end{abstract}

Keywords Thermal Stability, Reacting Slab, Hermite-PadÉ Approximation

\section{Introduction}

The study of thermaldeco mposition of reactive material in a slab is paramount in understanding the heat transfer of engineering processes. It is well known that thermal decomposition of different materials is dependent on size, shape and surface or environmental temperature as well as the physical properties of the material and environment. In other words for any given geometry, there is a critical size and surface temperature above which the heat generation inside the solid exceeds the heat dissipation to the surroundings [1].

Theoretical study of transient heating in a slab of combustible materialdue to exothermic chemical reaction plays a significant role in many industrial applications[1]. These include: heavy oil recovery, storage of cellulosic materials, the pyrolys is of bio mass and coal, the combustion of solids, waste incineration, coal gasification, etc. Without adequate knowledge of a reacting system, exothermic chemical process can accelerate significantly leading to runaway reaction, possible explosion, economical losses and cause emission of carbon dioxide and of toxic gases, like carbon monoxide through incomplete combustion ([2],[3],[4]). [5] presented a comprehensive review of chemical kinetic models for the heating-up of combustible materials. Meanwhile, analytical solutions of the highly nonlinear partial differential equations governing transient heating in a slab of combustible material due to exothermic reactions are usually impossible or extremely difficult to obtain. Hence in most cases, a numerical solution approach is adopted([6],[7],[8],[9],[10],[1])

\footnotetext{
* Corresponding author:

emassawe@uccmail.co.tz (Estomih S. Massawe)

Published online at http://journal.sapub.org/ijymp

Copyright (C) 2012 Scientific \& Academic Publishing. All Rights Reserved
}

There are serious challenges of dangerous reactive material which justify the analysis of thermal decomposition of reactive material in a slab. Because many facilities and systems have chemically reactive materials that may have serious consequences if they are not handled, used and stored properly. Previous studies of the thermal decomposition of reactive material dealt with different dimensional shapes like cylindrical, rectangular parallelepiped, sphere, etc. The model to be introduced in this study is based on studies of[2] and[3].[2] studied the dynamics of hollow material and investigated the problem of strong exothermic explosions in cylindrical pipe, for putting large activation energy and concluded that the procedure reveals accurately the steady thermal criticality condition.[3] studied hydrodynamics and investigated the effect of variable $v$ iscosity in a thermal decomposable generalized Newtonian fluid subjected to unsteady one dimension shear flow in which the result were presented for some parameters in the problem. It was observed that increasing the non-Newtonian nature of the flu id helps to delay the onset of thermal runaway when co mpared to Newtonian nature of the fluid.[4] studied the thermal decomposition in a slab and found that time-independent solutions for the spatial structure of temperature, considering a slab with isothermal boundaries subjected to exothermic reaction and uniform $p$ lastic heating.

Understanding the heat transfer and thermal stability characteristics of a reacting slab of combustive materials is extremely important in order to ensure the safety of its storage, handling and transportation([5,6]). Moreover, the phenomena of spontaneous ignition due to exothermic chemical kinetics in bulk solids such as coal, grain, hay, wool, etc., can be theoretically described by thermal combustion theory developed by[7,8]. One of the most important advantages of theoretical methods is that they can be applied as soon as a kinetic model had been evaluated fro $m$ data fro $m$ 
laboratory scale kinetic experiments. In particular, they allow estimation of runaway parameters in the earliest stages of the life cycle of a reactive material, thus ensuring elimination or significant reduction of the necessity for explosive experiments $([9,10])$.

In this present study, we investigate the heat transfer and thermal stability characteristics of a reacting slab of combustible material in the presence of heat loss, neglecting the reactant consumption. The associated nonlinear differential equation modelling the problem is tackled analytically using perturbation method together with a special type of Hermite-Padé series summation and improvement method $([11,12])$. Pertinent results are presented graphically and discussed.

\section{Mathematical Model}

Consider the steady-state of an exothermic chemical reaction in a slab of combustible material with possibility of heat loss to the surrounding ambient.

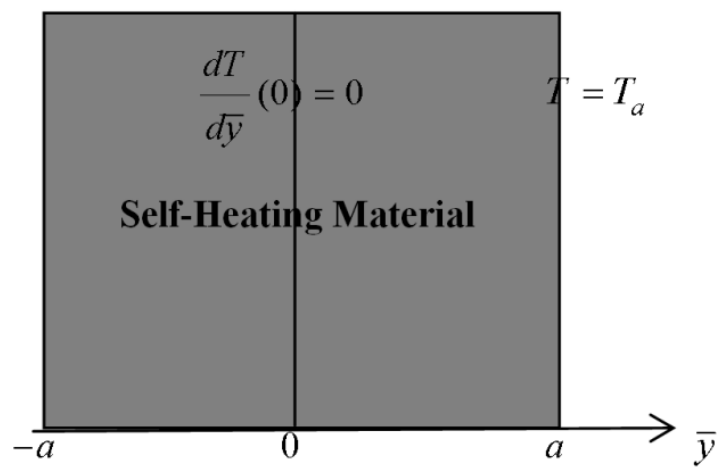

Figure 1. Geometry of the Problem

Assuming no reactant consumption, the one dimensional heat balance nonlinear partial differential equation governing the system given by $[5,9,10]$ is;

$$
\frac{d^{2} T}{d \bar{y}^{2}}+Q_{1} A C_{0}\left(\frac{K T}{v h}\right)^{m} e^{-\frac{E}{R T}}-\gamma\left(T-T_{a}\right)=0,
$$

with the in itial and boundary conditions as

$$
\frac{d T}{d y}(0)=0, T(a)=T_{a},
$$

where

$T$ is the absolute temperature,

$T_{a}$ is the ambient temperature,

$k$ is the thermal conductivity of the material,

$C_{0}$ is the reactant concentration,

$Q_{1}$ is the exothermicity,

$A$ is the rate constant,

$E$ is the activation energy,

$R$ is the universal gas constant,

$h$ is the Planck's number,

$K$ is the Boltzmann's constant,

$v$ is the vibration frequency,

$a$ is the slab half width, $\bar{y}$ is the distance measured transverse direction,

$\gamma$ is the heat loss parameter,

$m$ is the numerical exponent such that $m=\{-2,0,1 / 2\}$ represent numerical exponent for sensitized, Arrhenius and Bimolecular kinetics respectively.

We introduce the following dimensionless variables into equations.(1)-(2);

$$
\begin{gathered}
\theta=\frac{E\left(T-T_{a}\right)}{R T_{a}^{2}}, \quad \lambda=\frac{Q A E a^{2} C_{0}}{R T_{a}^{2}}\left[\frac{K T_{a}}{v h}\right]^{m} e^{-\frac{E}{R T_{a}}} \\
y=\frac{\bar{y}}{a}, \quad \varepsilon=\frac{R T_{a}}{E}, \quad \delta=\frac{R T_{a}^{2} \gamma}{Q A E C_{0}}\left[\frac{v h}{K T_{a}}\right]^{m} e^{\frac{E}{R T_{a}}},
\end{gathered}
$$

and we obtain the dimensionless governing equations as

$$
\frac{d^{2} \theta}{d y^{2}}+\lambda\left[(1+\varepsilon \theta)^{m} e^{\frac{\theta}{(1+\varepsilon \theta)}}-\delta \theta\right]=0,
$$

with

$$
\frac{d \theta}{d y}(0)=0, \theta(1)=0,
$$

where

$\lambda$ is the Frank-Kameneskii parameter,

$\varepsilon$ the activation energy parameter,

$\delta$ the heat loss parameter.

The thermal decomposition and stability of the reacting combustible material depend on the parameters in equation.(4), which are of great importance with respect to applications in the area of industrial safety and handling techniques of exp losives.

\section{Perturbation Method}

Due to the nonlinearity nature of the temperature variable in equations(4)-(5), it is convenient to seek a solution in the form of a power series expansion in parameter $\lambda$, i.e.

$$
\theta=\sum_{i=0}^{\infty} \theta_{i} \lambda^{i}
$$

Substituting the solution series in equation.(6) into equations.(4) and(5) and collecting the coefficients of like powers of $\lambda$, we obtained the followings:

Order zero $\left(\lambda^{0}\right)$

$$
\frac{d^{2} \theta_{0}}{d y^{2}}=0,
$$

with

$$
\frac{d \theta_{0}}{d y}(0)=0, \theta_{0}(1)=0
$$

Order one $\left(\lambda^{1}\right)$

$$
\frac{d^{2} \theta_{1}}{d y^{2}}=-\left(1+\varepsilon \theta_{0}\right)^{m} e^{\frac{\theta_{0}}{\left(1+\varepsilon \theta_{0}\right)}}+\delta \theta_{0},
$$

with

$$
\frac{d \theta_{1}}{d y}(0)=0,
$$


Order two $\left(\lambda^{2}\right)$

$$
\frac{d^{2} \theta_{2}}{d y^{2}}=-\left(1+\varepsilon \theta_{0}\right)^{m} e^{\frac{\theta_{0}}{\left(1+\varepsilon \theta_{0}\right)}}\left[\frac{\theta_{1}}{\left(1+\varepsilon \theta_{0}\right)^{2}}+\frac{m \varepsilon \theta_{1}}{\left(1+\varepsilon \theta_{0}\right)}\right]+\delta \theta_{1}
$$

with

$$
\frac{d \theta_{2}}{d y}(0)=0, \theta_{2}(1)=0
$$

and so on.

The above equations for the coefficients of solution series are solved iteratively for the temperature fields and reacting species concentration to obtain

$$
\begin{aligned}
& \theta(y)=-\frac{\lambda}{2}\left(y^{2}-1\right)-\frac{\lambda^{2}}{24}\left(y^{2}-1\right)\left(y^{2}-5\right)(-1-m \varepsilon+\delta) \\
& -\frac{\lambda^{3}}{720}\left(y^{2}-1\right)\left(-2 \delta y^{4}+8 m \varepsilon y^{4}+4 y^{4}-2 m \varepsilon y^{4} \delta-3 m \varepsilon^{2} y^{4}\right. \\
& -6 \varepsilon y^{4}+\delta^{2} y^{4}+4 m^{2} \varepsilon^{2} y^{4}-14 \delta^{2} y^{2}-52 m \varepsilon y^{2}+28 \delta y^{2}+12 m \varepsilon^{2} y^{2} \\
& -26 y^{2}+24 \varepsilon y^{2}-26 m^{2} \varepsilon^{2} y^{2}+28 m \varepsilon y^{2} \delta-66 \varepsilon+61 \delta^{2}+94+188 m \varepsilon \\
& \left.+94 m^{2} \varepsilon^{2}-122 m \varepsilon \delta-122 \delta-33 m \varepsilon^{2}\right)+O\left(\lambda^{4}\right)
\end{aligned}
$$

Using a computer symbolic algebra package(MAPLE), the first few terms of the above solution series in Eq.(6) are obtained. We are aware that these power series solutions are valid for very s mall parameter values of $\lambda$. However, using Hermite-Padé series summation and improvement technique, we have extended the usability of the solution series beyond small parameter values as illustrated in the following section.

\section{Thermal Stability Analysis}

From the application point of view, it is very important to determine the appearance of criticality or non-existence of steady-state solution for certain parameter values. In order to achieve this, a special type of Hermite-Padé series summation and improvement method will be utilized([11,12]). Let

$$
U_{N}(\lambda)=\sum_{n=0}^{N} a_{n} \lambda^{n}+O\left(\lambda^{N+1}\right), \text { as } \lambda \rightarrow 0,
$$

be a given partial sum. We note that equation(14) can be used to approximate any output of the solution of the problem under investigation, by employing Taylor expansion in a given small parameter. For instance, the series for the slab maximum temperature(i.e. $\theta_{\max }=\theta(0)$ along the slab centreline can be written as

$$
\begin{aligned}
& \theta(0)=\frac{\lambda}{2}-\frac{5 \lambda^{2}}{24}(-1-m \varepsilon+\delta)+ \\
& \frac{\lambda^{3}}{720}\left(94-66 \varepsilon+61 \delta^{2}+188 m \varepsilon+94 m^{2} \varepsilon^{2}\right. \\
& \left.-122 m \varepsilon \delta-122 \delta-33 m \varepsilon^{2}\right)+O\left(\lambda^{4}\right)
\end{aligned}
$$

Let us assume that $U(\lambda)$ is a local representation of an algebraic function of $\lambda$ in the context of nonlinear problems. We seek an expression of the form

$$
F_{d}(\lambda, U)=\sum_{m=1}^{d} \sum_{k=0}^{m} f_{m-k, k} \lambda^{m-k} U^{k}
$$

of degree $d \geq 2$, such that

$$
\frac{\partial F_{d}}{\partial U}(0,0)=1 \text { and } F_{d}\left(\lambda, U_{N}\right)=O\left(\lambda^{N+1}\right)
$$$$
\text { as } \lambda \rightarrow 0
$$

The requirement (17) reduces the problem to a system of $N$ linear equations for the unknown coefficients of $F_{d}$. The entries of the corresponding matrix depend only on the $N$ given coefficients $a_{n}$ and we shall take $N=\frac{1}{2}\left(d^{2}+3 d-2\right)$, so that the number of equations equals the number of unknowns. The polynomial $F_{d}$ is a special type of Hermite-Padé approximant and is then investigated for bifurcation and criticality conditions using Newton dia$\operatorname{gram}([13])$.

\section{Results and Discussion}

In this section, we validate the above theoretical results using physically realistic values of various embedded parameters in the numerical experiment. We note that increasing parameter value of $\lambda$ indicates an increase in the rate of exothermic chemical kinetics in the slab. In order to obtain the thermal stability criterion in the reacting slab, the Hermite-Padé approximation procedure in section(4) above was applied to the first few terms of the solution series in section(3) and we obtained the results as shown in tables(1) and(2) below:

The results in table(1) reveal the rapid convergence of Hermite-Padé series summation and improvement procedure with gradual increase in the number of series coefficients utilized in the approximants for the thermal criticality condition. In table (2), it is noteworthy that the magnitude of thermal explosion criticality $\left(\lambda_{c}\right)$ increases with an increase in the parameter values of $\beta>0$ due to an increase in the heat loss to the surrounding ambient. This invariably will lead to a de lay in the develop ment of thermal runaway in the reacting slab. Since the heat is not accumulated in the slab, it enhances the thermal stability of the system. Similar effect of thermal stability enhancement is observed with increasing values of activation energy parameter $(\varepsilon)$. Increasing values of $\varepsilon$ implies that the activation energy of the reacting slab is very low; hence the volatility tendency of the reacting slab is greatly reduced. Furthermore, it is interesting to note from table(2) that thermal explosion occur faster in a bimolecular $(m=0.5)$ type of exothermic reaction as compared to the Arrhenius $(m=0)$ and sensitised $(m=-2)$ type of reaction. A slice of the bifurcation diagram for $0 \leq \varepsilon \ll 1$ in the $\left(\lambda, \theta_{\max }\right)$ plane is shown in figure.(2). It represents the qualitative change in the thermal system as the parameter $(\lambda)$ increases. In particular, for $0 \leq \varepsilon \ll 1$ and $\delta>0$ there is a critical value $\lambda_{c}$ (a turning 
point) such that, for $0<\lambda<\lambda_{c}$ there are two solutions(labelled I and II). The upper and lower solution branches occur due to the nonlinearity in the energy balance equation. When $\lambda>\lambda_{c}$ the system has no real solution and displays a classical form indicating thermal runaway. As exothermic reaction due chemical kinetics increases, the slab temperature increases uncontrollably until it ignites.

Table 1. Computations Showing the Criticality Procedure Rapid Convergence $(m=0, \delta=\varepsilon=0.1)$

\begin{tabular}{|c|c|c|c|}
\hline$d$ & $N$ & $\theta_{\max }$ & $\lambda_{c N}$ \\
\hline 2 & 4 & 1.534462519 & 1.0294760324 \\
\hline 3 & 8 & 1.526450745 & 1.0294762373 \\
\hline 4 & 13 & 1.523940782 & 1.0294759734 \\
\hline 5 & 19 & 1.523941684 & 1.0294759811 \\
\hline 6 & 26 & 1.523941684 & 1.0294759811 \\
\hline
\end{tabular}

Table 2. Computations Showing Thermal Explosion Criticality for Different Parameter Values

\begin{tabular}{|c|c|c|c|c|}
\hline$\delta$ & $m$ & $\varepsilon$ & $\theta_{\max }$ & $\lambda_{c}$ \\
\hline 0 & 0.0 & 0.1 & 1.524355912 & 0.98820780 \\
\hline 0.1 & 0.0 & 0.1 & 1.523941684 & 1.029475981 \\
\hline 0.5 & 0.0 & 0.1 & 1.521889849 & 1.235982214 \\
\hline 1.0 & 0.0 & 0.1 & 1.517878271 & 1.649946712 \\
\hline 0.1 & 0.5 & 0.1 & 1.419877455 & 0.968854619 \\
\hline 0.1 & -2.0 & 0.1 & 2.221630082 & 1.387841844 \\
\hline 0.1 & 0.0 & 0.01 & 1.211934332 & 0.920984440 \\
\hline 0.1 & 0.0 & 0.0 & 1.186529145 & 0.910930564 \\
\hline
\end{tabular}

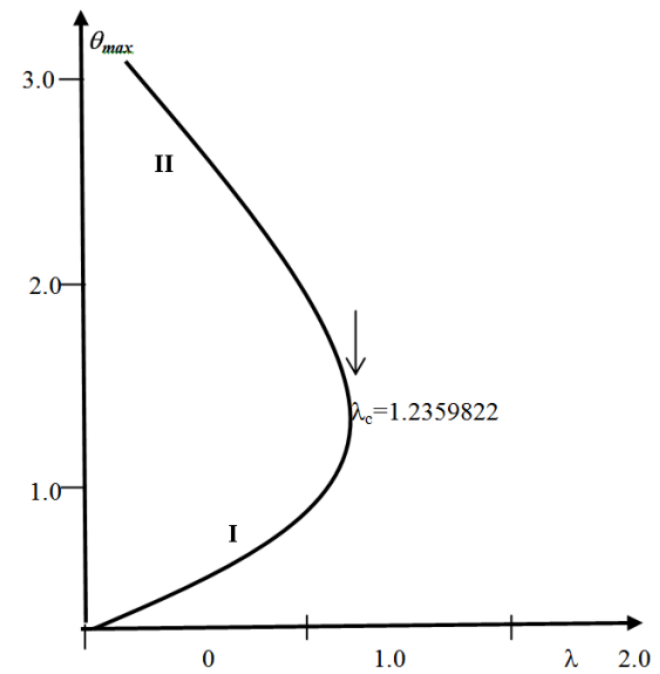

Figure 2. A Slice of Approximate Bifurcation Diagram in the $\left(\lambda, \theta_{\max }\right)$ PlaneWhen $\delta=0.5, \varepsilon=0.1, m=0$

Figures(3)-(5) illustrate the effects of various thermophysical parameters on the steady state slab temperature profiles. The temperature is maximum along the slab centerline and minimu $\mathrm{m}$ at the slab surface. Figure(3) shows that the slab temperature is highest during bimolecular reaction $(m=0.5)$ and lowest for sensitized reaction $(m=-2)$, hence confirming the earlier results in the literature. This observation is also in agreement with the results highlighted in table(2). In figure(4) we observe that the slab temperature decreases with an increase in the heat loss parameter $\delta$. The decrease in the slab temperature with increasing $\delta$ can be attributed to the cooling action of heat loss on the slab. Figure(5) show that the slab temperature increases with an increase in the parameter values of $\lambda$. As the Frank-Kamenetskii parameter $(\lambda)$ increases, the slab internal heat generation due to exothermic reaction increases, this invariably leads to an elevation in the slab temperature.

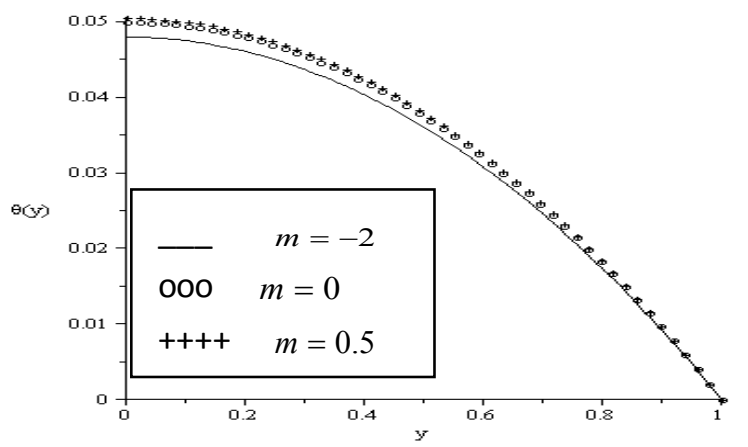

Figure 3. Effect of Reaction Type Index on Temperature Profiles $\lambda=0.1, \delta=0.1, \varepsilon=0.5$

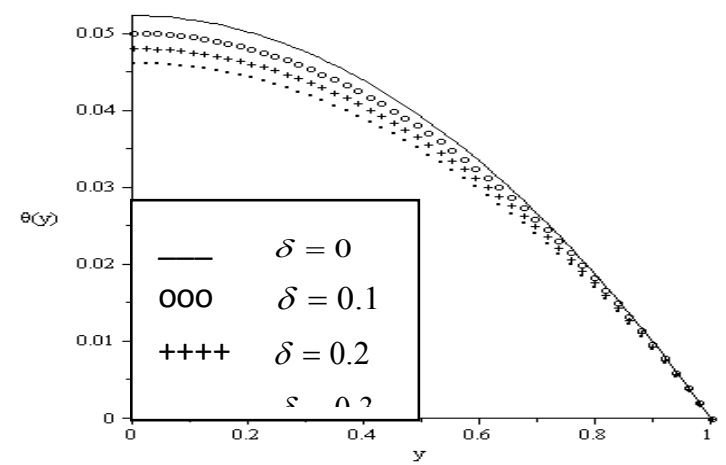

Figure 4. Effect of Heat Loss on Slab Temperature Profiles for $m=0.5, \lambda=0.1, \varepsilon=0.1$

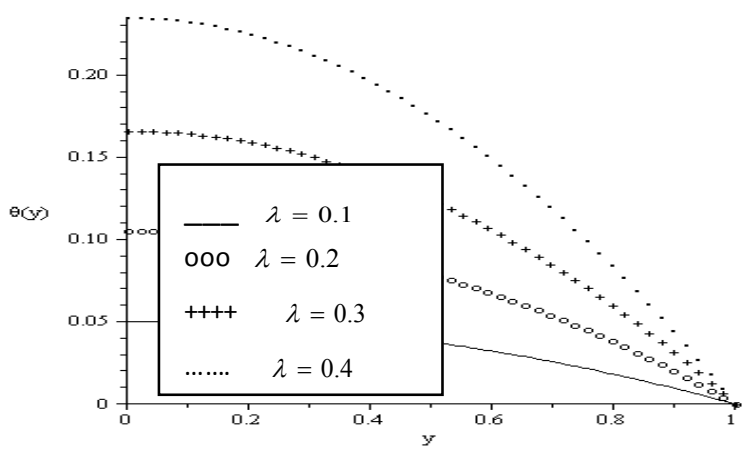

Figure 5. Effect of Increase in Exothermic Reaction Rate on the Slab Temperature Profiles for $m=0.5, \delta=\varepsilon=0.1$

\section{Conclusions}

The steady state exothermic chemical reaction in a slab of combustible material is considered. The nonlinear ord inary differential equation governing the problem was formulated and solved analytically using the perturbation technique together with a special type of the Hermite-Padé series 
summation and improvement method. The possibility of thermal runaway phenomena was shown and the corresponding thermal criticality values were obtained and illustrated on a bifurcation diagram. An increase in the parameter value of $\lambda$ indicates increasing rate of exothermic chemical kinetics. As parameter $\delta$ increases in value $(\delta>0)$, the rate of heat loss to the ambient increases as well as the thermal explosion criticality values. This invariably enhances the thermal stability of the reacting slab by preventing the occurrence of thermal runaway.

\section{REFERENCES}

[1] Lohrer C., Schmidt M., Krause U., A study on the influence of liquid water and water vapour on the self-ignition of lignite coal - experiments and numerical simulations, Journal of Loss Prevention in the Process Industries, 18(3), pp. 167-177, 2005.

[2] Balakrishnan E, Swift A, Wake GC . Critical values for some non-class A geometries in thermal ignition theory. Math. Comput. Modell. 24, pp. 1-10, 1969

[3] Kamenetskii DAF (1969). Diffusion and heat transfer in chemical kinetics. Plenum Press, New York, 1969.

[4] Warnatz J, Maas U, Dibble R., Combustion: Physical and chemical fundamentals, Modelling and simulation, Experiments, Pollutant formation, Springer-Verlag Berlin and Hiedelberg $\mathrm{GmbH}$ and Co. K., 2001.

[5] Simmie J.M., Detailed chemical kinetic models for the combustion of hydrocarbon fuels, Prog. Energy Combust. Sci., 29, pp. 599-634, 2003.

[6] Makinde O. D., Exothermic explosions in a slab: A case study of series summation technique, International Communications in Heat and Mass Transfer, Vol. 31, No.8, pp. 1227-1231, 2004.

[7] Sadiq M. A., Merkin J. H., Combustion in a porous material with reactant consumption: The role of ambient temperature. Mathl Comput. Modelling, 20, 27-46, 1994

[8] Bebernes J, Eberly D., Mathematical problems from combustion theory. Applied Mathematics Series, vol. 83, Springer-Verlag, New York, Inc. 177, 1989.

[9] Makinde O.D., Moitsheki R.J., Group method solution of nonlinear heat diffusion model for thermal energy storage problem, International Journal of Physical Sciences Vol. 5(3), 246-250, 2010.

[10] Phiri P. A, Makinde O. D., A new derivative free method for solving nonlinear equations. International Journal of the Physical Sciences, Vol. 5, No. 7, 935-939, 2010.

[11] Legodi A. M. K., Makinde O. D., A numerical study of steady state exothermic reaction in a slab with convective boundary conditions. International Journal of the Physical Sciences, Vol. 6(10), 2541-2549, 2011.

[12] JShouman A.R., A very simple yet accurate solution to the thermal explosion problem, Journal of loss Preservation in the Process Industries, Vol.11, pp. 383-390, ELSEVIER, 1998.

[13] Makinde O .D, Strong exothermic explosions in a cylindrical pipe: A case Study of series summation technique, Mechanical Research Communications, Vol. 32, pp. 191-195, ELSEVIER, 2005

[14] Bankole A.O., Thermal decomposition in ID Shear Flow of Generalized Newtonian Fluids. African Institute for Mathematical Sciences (AIMS), 2009.

[15] Dienes J. K., On reactive shear bands, Phys. Lett. A 118,pp. 433-438, 1986.

[16] Williams F. A., Combustion theory. Second Edition, Benjamin \& Cuminy publishing Inc. Menlo Park, Califonia, 1985.

[17] Frank-kameneskii D.A., Diffusion and heat transfer in chemical kinetics, Plenum Press, New York, 1969.

[18] ]Semenov N. N., Some problems in Chemical kinetics and Reactivity, Princeton University press, Princeton, 1956.

[19] Balakrishnan E., Swift A., Wake G. C., Critical values for some non-class A geometries in thermal ignition theory. Math. Comput. Modell. 24, pp. 1-10, 1996.

[20] Sergeyev A. V., Goodson D. Z., Summation of asymptotic expansions of multiple valued functions using algebraic approximations-application to anharmonic oscillators, J. Phys. A31, pp. 4301-4317, 1998.

[21] Makinde O. D., Solving microwave heating model in a slab using Hermite-Pade approximation technique, Applied Thermal Engineering, Vol. 27, pp. 599-603, 2007.

[22] Vainberg M. M., Trenogin V. A., Theory of branching of solutions of nonlinear equations, Noordoff, Leyden, 1974 